\title{
DELEUZE Y LA IMPORTANCIA DE HUME. UNA LECTURA OLVIDADA*
}

\author{
Myriam Hernández Domínguez \\ Universidad de La Laguna \\ mhernado@ull.edu.es
}

\section{RESUMEN}

Son recurrentes las reflexiones sobre la influencia de Spinoza, Bergson o Nietzsche en el pensamiento de Gilles Deleuze, pero ¿qué ocurrió con Hume, a quien Deleuze dedicó una de sus primeras obras? La intención de este artículo es mostrar la lectura que hace Deleuze de Hume en Empirismo y subjetividad y la relevancia que tiene la interpretación del hábito en uno de sus libros más importantes, Diferencia y repetición.

Palabras clave: Deleuze, Hume, hábito, diferencia, repetición.

\section{DELEUZE AND THE IMPORTANCE OF HUME. A FORGOTTEN READING}

\section{Abstract}

Reflections on the influence of Spinoza, Bergson or Nietzsche on Gilles Deleuze's thinking are recurrent, but what happened to Hume, to whom Deleuze dedicated one of his first works? The intention of this article is to show Deleuze's reading of Hume in Empiricism and Subjectivity and the relevance of the interpretation of the habit in one of his most relevant books Difference and Repetition.

Keywords: Deleuze, Hume, habit, difference, repetition. 
Hume es un desvelador de secretos para Deleuze. Si bien a lo largo de la historia de la filosofía se ha definido el empirismo como lo inverso al racionalismo y opuesto al innatismo, según el filósofo francés, el empirismo siempre ha tenido otros secretos, y presenta a Hume como el filósofo que ha arrojado luz sobre ellos. Será en su primer texto, Empirismo y subjetividad, publicado en 1953, donde Deleuze los aborde al ritmo de una pregunta central en la filosofía humeana: ¿cómo la mente deviene naturaleza humana? o ¿̇cómo la mente deviene sujeto? En Diferencia y repetición se puede observar gran cantidad de vínculos y conexiones con Empirismo y subjetividad. Deleuze recupera el conjunto de análisis sobre Hume realizados en Empirismo y subjetividad para fundamentar su concepción de la repetición. En las siguientes páginas trataré de manifestar qué hay de una obra en la otra. Los primeros apartados de este trabajo abordan la lectura que hace Deleuze de Hume en la citada obra, para, finalmente, dilucidar la importancia que tienen sus reflexiones en Diferencia y repetición.

\section{HÁBITO, EXPERIENCIA E IMAGINACIÓN. CONCEPTOS CLAVE}

La naturaleza humana es la única ciencia del hombre posible para Hume. Esta debe ser estudiada no científicamente, sino en los efectos sobre la mente. Hume presentará dos modalidades de esta ciencia, una suerte de dos géneros de afección. Por un lado, los efectos de asociación y, por otro, los efectos de la pasión. Cada una de ellas es la determinación de un sistema; el del entendimiento, el de las pasiones o el de la moral. La asociación, por su parte, le otorga a la mente una generalidad necesaria, mientras que la pasión dota a la mente de una constancia, posibilitando una actividad práctica y moral. La ausencia de este doble movimiento supone, por una parte, la ausencia de una naturaleza humana y, por otra, que la imaginación siga siendo una fantasía. Esto no supone que haya que identificar el sistema del entendimiento con la teoría y el de moral con la práctica. Hume apuesta por una práctica del entendimiento y por una teoría moral en relación con la organización social y política: «Para Hume la única teoría posible es una teoría de la práctica»"

De este modo, es posible rescatar el problema de la razón. Es preciso percatarse, mantiene Deleuze, de que el problema de la razón se da en un dominio que escapa a la razón misma y la pone en cuestión. La influencia de la razón en la práctica se hace latente en la comunicación de la existencia de un objeto propio de una pasión, una cosa, mostrándonos una conexión de causas y efectos. No obstante, no es posible afirmar que la razón genere una acción, ni que combata una pasión, ni que esta la contradiga ${ }^{2}$. La razón tampoco genera las distinciones morales, estas

* Este trabajo ha sido desarrollado gracias al apoyo de la Ayuda para la Formación del Profesorado Universitario (FPU) financiada por el Ministerio de Ciencia, Innovación y Universidades de Gobierno de España. Referencia personal: FPU2017/5003.

${ }^{1}$ Deleuze, G., Empirismo y subjetividad, Gedisa, Barcelona, 2015, p. 24.

2 Ibidem, p. 25. 
son las que despiertan las pasiones y la producción o impedimento de la acción. La razón, entonces, se aplica siempre a un mundo que la precede. Esto quiere decir que la práctica y la moral, en su naturaleza, son diferentes a la razón.

La razón es la imaginación que ha devenido naturaleza, el conjunto de los efectos simples de la asociación, ideas generales, substancias, relaciones ${ }^{3}$. Dado que existen dos tipos de relaciones habrá que distinguir dos tipos de razones. Según Hume es posible diferenciar entre las relaciones de ideas y las relaciones de objetos. Las primeras dependen de la comparación entre las ideas, en términos, por ejemplo, de semejanza, grados de cualidad, contrariedad, etc. Las segundas no dependen de las ideas, como ocurre con las relaciones de identidad o de tiempo y lugar. Así, es posible distinguir dos razones: la que procede por certidumbre (intuición o demostración) y la que procede en función de probabilidades (razón experimental o entendimiento). La experiencia y el hábito son los dos principios fundamentales de la razón experimental, la naturaleza humana pasa por el rodeo de una observación de la naturaleza, de una experiencia de la naturaleza ${ }^{4}$. El hábito y la experiencia son principios de la naturaleza que se forman progresivamente; se unen para actuar sobre la imaginación.

Sin embargo, hábito y experiencia son principios distintos. La experiencia, por su parte, incide en la observación de conjunciones particulares, en la repetición de casos parecidos. Su efecto, apunta Deleuze, es la causalidad como relación filosófica: la imaginación deviene un entendimiento. Sin embargo, esa repetición de casos particulares no forma nada, no es una progresión, no nos hace avanzar. Es preciso, entonces, que el entendimiento extraiga, de un principio distinto a la experiencia, la capacidad de inferir, es decir, de sacar, superar, y extraer conclusiones de la experiencia. Este principio es el hábito que, como podemos intuir, está al margen de una lógica de la cantidad, tratando de no limitarse a repetir casos que no pretendan alcanzar una idea nueva. El efecto del hábito cobra importancia en el hecho de que la imaginación deviene creencia porque se produce una transición de la impresión de un objeto a la idea de otro. Esta idea se vincula con la que, para Deleuze, es una de las partes que conforman la doble implicación del hábito. Por un lado, el hábito permite al entendimiento razonar sobre la experiencia al hacer de la creencia un acto posible del entendimiento. Por otro, presupone la experiencia, pues los objetos se unen en la imaginación porque se da un descubrimiento previo de la conjunción de los objetos 5 . Así, la repetición se hace progresión y producción, al ser conside-

3 Ibidem, pp. 26-29.

4 «Puesto que, según el propio Hume, las relaciones de los objetos pueden modificarse sin el más mínimo cambio en las ideas mismas, es decir, puesto que, consideradas en sí mismas, las ideas de los objetos pueden estar vinculadas entre sí por cualquier relación, es necesario recorrer cada vez el camino que lleva de la idea a la impresión, para determinar qué relaciones están dictadas en este caso por la experiencia y el hábito que son los dos principios fundamentales del razonamiento experimental». Martelaere, P., "Deleuze, interprète de Hume», en Revue Philosphique de Louvain, n. ${ }^{\circ}$ 54, pp. 224-248. [La traducción es mía].

5 Ibidem, pp. 66-69. 
rada dentro de la mente que la contempla y productora de una nueva impresión, y no como una mera repetición de objetos en los que no descubre ni produce nada. La repetición produce en la mente no solo una nueva impresión, sino, también, una espera y una tendencia.

El mundo de la moral (artificio) y el mundo del conocimiento (hábito) se encuentran en el origen de las reglas generales que son, a la par, extensivas y correctivas. En el mundo de la moral, las reglas generales se refieren a la reflexión de los principios de la naturaleza en la imaginación. En el sistema del conocimiento las reglas generales aluden al carácter particular de un principio. Esto supone, no solo una experiencia, sino, también, una formación. La idea en la que creemos se encuentra vinculada a una impresión que fija, en la imaginación, la idea a la que la impresión comunica su vivacidad. En este proceso comunicativo participan la semejanza y la contigüidad, pero también la causalidad, es decir, el hábito ${ }^{6}$ en tanto que repetición de los casos de conjunción constante observados en la experiencia entre dos objetos.

Sin embargo, el hábito por sí mismo puede invocar una falsa experiencia, generando una creencia a partir de una repetición que no parte de la experiencia: una creencia ilegítima, una ficción de la imaginación, según Deleuze. De este modo, la imaginación, aunque se debe fijar por el principio del hábito, también se sirve de él para superar la ficción, para desbordar la experiencia. Las creencias que son generadas de este modo son denominadas «ilegítimas» desde el ejercicio del entendimiento, pero también «inevitables», pues forman las reglas generales extensivas y desbordantes. Estas creencias ilegítimas, así como las repeticiones que no proceden de la experiencia, tienen el lenguaje y la fantasía como fuentes principales. El lenguaje sustituye la repetición observada por la hablada, creando una creencia. El filósofo, dice Hume, a fuerza de hablar de facultades y cualidades ocultas, termia por creer que las palabras tienen un sentido oculto que podemos descubrir gracias a la reflexión ${ }^{7}$. La fantasía, por su parte, nos lleva a confundir lo accidental con lo esencial. La falsificación de las creencias dependerá siempre de lo accidental, de las disposiciones de la persona. Por esto, podemos afirmar que la imaginación es desbordante tanto en el sistema del entendimiento como en el sistema de la moral. En el caso del conocimiento, se encuentra en la negatividad del error y, por ello, debe combatirse a partir de la corrección, entendida como denuncia de un error. Es decir, puesto que la imaginación no cree sin falsificar la experiencia $-y$, por tanto, el hábito falsifica la experiencia e invoca a las repeticiones falsas-, es precisa la existencia de una corrección, de una reflexión posterior, de una suerte de reglas que permitan distinguir lo general de lo accidental. Tratar de mantener la creencia en los límites del entendimiento y, por ende, hacer que el hábito se corresponda con la experiencia es el objetivo de la probabilidad filosófica. De este modo, es posible entender la máxima de

\footnotetext{
${ }^{6}$ «... Hume siempre da dos definiciones conjuntas de la causalidad: unión de objetos semejantes e inferencia de la mente de un objeto a otro». Ibidem, p. 69.

7 Ibidem, p. 70.
} 
Hume: «Para que el razonamiento sea legítimo es preciso que nazca del hábito, no directamente sino oblicuamente» ${ }^{8}$.

\section{SUJETO, HÁBITO Y RELACIONES. LA TRANSFORMACIÓN DE LA MENTE}

Retomo la pregunta con la que iniciaba el primer punto, la cuestión del sujeto. Deleuze responde de manera clara: «Cuando hablamos de sujeto queremos decir que la imaginación, de simple colección que era, pasa a ser una facultad ${ }^{9}$, es decir, la colección se convierte en sistema. Atendiendo a la terminología de Hume, lo que ocurre es que la mente se convierte en naturaleza humana, supera lo dado. La mente es esencialmente sucesión, mientras que el sujeto es duración, duración de un hábito, de una espera ${ }^{10}$. El juego entre hábito y espera es fundamental para Deleuze y la construcción de su propia filosofía, pues el hábito es espera, y viceversa, lo que implica un dinamismo entre el ímpetu hacia el futuro y el empuje del pasado que aparece ya, admirablemente, en la filosofía de Hume. El hábito, anuncia Deleuze, es la raíz constitutiva del sujeto y lo que el sujeto es, en su raíz, es la síntesis del tiempo: del presente y del pasado en miras al porvenir: ${ }^{11}$

No se pude decir mejor que el tiempo se halla en una relación tal con el sujeto, que el sujeto nos presenta la síntesis del tiempo, y que ésta es únicamente productiva, creadora, inventiva ${ }^{12}$.

La síntesis, por tanto, puede ser entendida como el planteamiento del pasado, como regla del porvenir. El tiempo era estructura, explica Deleuze, y el sujeto se presenta como síntesis del tiempo. Para entender esta transformación es necesario evocar a la memoria, pues la mente implicaba la memoria. La memoria, entendida por Hume, es la reaparición de una impresión en forma de una idea viva. Hume distinguiría, en la colección de percepciones, entre idea y memoria y entre impresiones y sentidos, según grados de vivacidad. Es evidente, a primera vista, dirá Hume, que las ideas de la memoria son mucho más vivaces y consistentes que las de la imaginación, y que la primera facultad nos presenta sus objetos más exactamente de lo

8 Ibidem, p. 73.

9 Ibidem, p. 100.

10 «El principio del hábito en tanto que fusión de los casos semejantes en la imaginación, y el principio de conocimiento, se combinan así para producir a la vez la relación y la inferencia de acuerdo con la relación (creencia), y ambos constituyen los principios según los cuales funciona la causalidad». Ibidem, p. 216.

${ }^{11}$ Esta afirmación se encuentra en Empirismo y subjetividad. Como veremos en las siguientes páginas, este razonamiento será expresado, con insistencia, quince años más tarde, en Diferencia y repetición.

12 Ibidem, p. 102. 
que lo hace la última (2001: 25$)^{13}$. Sin embargo, la memoria solo es capaz de reproducir las estructura de lo dado, no de superarlas, y, por tanto, tampoco será capaz de operar la síntesis. El hábito será, entonces, quien opere la síntesis. De este modo, es posible entender la sentencia del Deleuze: «El hábito es a la memoria lo que el sujeto es a la mente» (2015: 103). El hábito no precisa de la memoria en tanto que no necesita evocar ningún recuerdo, el pasado como tal no es dado, es construido por una síntesis que es, a su vez, fuente y origen del sujeto.

En este punto, Deleuze plantea que, si bien en la práctica no existe problema alguno en la comprensión de la síntesis, pues nos damos en el pasado y en el presente, efectuándose la síntesis por sí sola, hay que percatase de que el presente y el pasado no son caracteres del tiempo. Es decir, el pasado y el presente ${ }^{14}$ se constituyen en el tiempo siendo afectados por otros principios. En este sentido, la síntesis misma no es más que esa esa organización, esa «doble afección» que llama Deleuze. Entender, por tanto, cómo se constituyen en el tiempo un presente y un pasado pasa por regresar a Hume y a dos de sus principios: la experiencia y el hábito ${ }^{15}$. La experiencia, por su parte, es una multiplicidad, implica la repetición de casos semejantes. La experiencia afecta al tiempo con un pasado, refleja el tiempo como pasado sometido a la observación. El hábito organiza el tiempo como presente perpetuo al que hay que adaptarse, refleja el tiempo como porvenir lleno de esperas. De este modo, entonces, se transforma el tiempo cuando el sujeto se transforma en mente.

Si ya sabemos cómo se transforma el tiempo cuando el sujeto se constituye en la mente, la pregunta que aún le queda a Deleuze por plantear es ¿qué ocurre con el organismo que, hasta ahora, había sido concebido como mecanismo de percepciones distintas? El organismo adquiere, según Deleuze, una «doble espontaneidad». Por un lado, una espontaneidad de la relación, que se refiere al hecho de despertar a las ideas vinculadas cuando se percibe una idea. Para que esto ocurra es necesario que las ideas estén asociadas en la mente, es decir, se precisa que el mecanismo de percepciones distintas esté recortado en el cuerpo mismo por una espontaneidad física de las relaciones. Hablamos, por tanto, de una espontaneidad del cuerpo que depende de los mismos principios que la subjetividad ${ }^{16}$. El cuerpo, por tanto, ha pasado de ser mera mente, colección de ideas e impresiones, a ser el sujeto considerado en esa espontaneidad de las relaciones que establece, influenciado por los principios, entre las ideas. Por otra parte, la espontaneidad de disposición se refiere a la inclinación propia y particular del organismo a la producción de una pasión. Es decir, la disposición, a partir de la pasión, genera espontáneamente una idea, una idea del objeto

${ }_{13}$ Hume, D., Tratado de la naturaleza humana, Editorial Nacional, Madrid, 2001, p. 114.

${ }_{14}$ Deleuze puntualiza, casi en la mitad de su obra, algo esencial, ¿qué entiende por presente y pasado?: «El presente y el pasado -comprendido uno como la partida de un impulso, y el otro como el objeto de una observación- no son caracteres del tiempo» (2015: 103).

15 «La experiencia es la dimensión retrospectiva que da una estructura objetiva al presente a partir de la observación de los casos en el pasado; el hábito es la visión prospectiva que sintetiza esos casos, generalizándolos, en función de observaciones posibles en el futuro» (2016: 74).

16 Ibidem, p. 105. 
que responde a la pasión. En este sentido, el cuerpo es la fuente biológica de la espontaneidad a la que remiten las impresiones de reflexión que constituyen al sujeto.

Pero ¿qué principios son los que constituyen al sujeto en la mente? Recordemos que lo que transforma la mente es el sujeto, y lo que constituye al sujeto son los principios de asociación y los principios de la pasión. Si bajo los principios de asociación se establecen las relaciones entre ideas, bajo los de la pasión el sujeto persigue un fin organizando los medios de los que dispone ${ }^{17}$. Lo que pretende Deleuze, recuperando la pregunta por la constitución del sujeto, es poner el énfasis en las relaciones. $\mathrm{Si}$ bien las relaciones no dependen de las ideas, las causas de la relación determinan al sujeto, que es el único capaz de establecer relaciones.

Existen dos especies de relaciones. La primera de ellas se refiere a la relación de un objeto variable con el conjunto en el que se integra. Esto es lo que nos presentan las relaciones de espacio y tiempo bajo distintas formas: distancia, contigüidad, anterioridad, etc. Si la mente por sí misma ya nos daba las nociones de distancia y contigüidad, lo que explica es el hecho de que la contigüidad y las distancia sean relación. En este sentido, el espacio y el tiempo no eran en la mente más que una composición ${ }^{18}$. Y ¿cómo llegan a ser una relación? Aquí aparece la segunda especie de relación, la relación, en este caso, es ficción: se aplica la idea de tiempo a un objeto invariable y se comparan las representaciones de ese objeto con el conjunto de nuestras percepciones. Esta segunda especie de relaciones pone en relación los caracteres de dos o más ideas que han sido consideradas individualmente. En este sentido, es posible afirmar que las relaciones no cambian sin que lo hagan las ideas; la materia de la comparación es una idea distinguible. Sin embargo, las relaciones son siempre exteriores, siempre suponen una síntesis, acerca de la cual ni la idea ni la mente pueden informar.

Las relaciones son efectos de los principios de asociación (contigüidad, semejanza y causalidad) que dan constancia a la mente. A pesar de que cada uno de ellos se dirija a un aspecto concreto de la mente -la contigüidad a los sentidos, la semejanza a la imaginación y la causalidad al tiempo-, tienen un punto común; designar una cualidad que conduce a la mente de una idea a otra ${ }^{19}$. Bajo el efecto de los principios de asociación es posible, entonces, comparar, agrupar y evocar ideas. Es decir, el efecto de los principios de asociación son las ideas complejas, a saber, las relaciones, modos o ideas generales. Al mismo tiempo que a la mente se le designan ideas, se convierte en sujeto. Esta tesis sirve para reforzar la idea que destaca Deleuze sobre las relaciones; que son externas a sus términos quiere decir que las ideas no informan sobre la naturaleza de las operaciones que se efectúan a partir de ellas, sino que los

17 «Si los principios de asociación son exteriores a las ideas, las relaciones, que son el resultado de la asociación, son obviamente también externas. Deleuze ve en la aceptación implícita de esta presuposición no sólo la esencia de la filosofía de Hume, sino el punto de partida común para todas las formas de empirismo». Martelaere, P., op. cit., p. 228. [La traducción es mía].

18 Ibidem, p. 109.

19 Recordemos que esta cualidad de introducir naturalmente otra idea distinta no es una cualidad de la idea en sí, sino de la naturaleza humana. 
principios de la naturaleza humana (los principios de asociación) son la condición necesaria de las relaciones. Sin embargo, apunta Deleuze, la asociación no basta para explicar las relaciones, solo las posibilita ${ }^{20}$. La semejanza, por tanto, no siempre produce una asociación de ideas. Aparece aquí la gran objeción al asociacionismo: los principios de asociación explican la forma del pensamiento general pero no los contenidos singulares. Deleuze insiste en que tanto Bergson como Freud están de acuerdo en este punto, la asociación solo explica la superficie de nuestra consciencia, «la corteza». Sin embargo, es necesario señalar que Hume fue un pionero en pensar en esto; la asociación de ideas informa sobre los hábitos del pensamiento, las ideas corrientes o de las nociones cotidianas del buen sentido, pero la asociación de ideas no explica la diferencia entre una mente y otra, es decir, no explica que se evoque una idea antes que otra:

Desde este punto de vista, hay que definir la relación como esa particular circunstancia por la cual juzgamos bueno comparar dos ideas, aun cuando estas se encuentren arbitrariamente unidas en la imaginación. Si bien es cierto que la asociación es necesaria para posibilitar toda relación en general, en modo alguno la asociación explica cada relación en particular ${ }^{21}$.

Lo que dotará a la relación de su razón será la circunstancia que, según Hume, es la que hace posible la ciencia de lo particular. Para Hume, la noción de circunstancia designa a la afectividad; «la afectividad es asunto de las circunstancias». Las circunstancias, entonces, en tanto que representan un estado de las pasiones y las necesidades, singularizan al sujeto. Por tanto, los principios de asociación explican que las ideas se asocian, y los principios de la pasión que determinada idea se asocie, en un momento concreto, antes que a otra idea. En síntesis, lo que pretende poner de manifiesto Deleuze es que el sujeto se presenta en la mente bajo los efectos de dos principios que se conjugan; los principios de asociación, que dotan al sujeto de su forma necesaria, y los principios de la pasión, un contenido singular. Esta unidad entre principios se expresa en la mente a través del vínculo entre relación y circunstancia que he tratado de explicar. Es decir, si la relación no se separa de las circunstancias, esto es, si el sujeto no puede separase del contenido singular que le es esencial, la subjetividad es esencialmente práctica. El sujeto se constituye en lo dado.

${ }^{20}$ Hume denominará «relación natural» a lo que la asociación explica y «relación filosófica» a lo que no puede explicar.

${ }_{21}$ Ibidem, p. 113. 


\section{DIFERENCIA Y REPETICIÓN. LA INFLUENCIA DE HUME O EL HÁBITO COMO PRIMERA SÍNTESIS TEMPORAL}

La influencia de Hume en Diferencia y repetición se hace visible en el segundo concepto de la disyunción. La repetición y, concretamente, la noción de hábito tratada por Deleuze pone de manifiesto la influencia de la lectura humeana en su obra. En este punto me ocuparé de poner de manifiesto lo que puede haber de Empirismo y subjetividad en Diferencia y repetición con respecto a la noción de hábito.

La repetición no es la generalidad. Esta es la primera sentencia de Deleuze sobre su comprensión de la repetición. Existe una diferencia de naturaleza entre ambos conceptos, pues, si bien la generalidad se caracteriza por el intercambio o sustitución de algo particular, la repetición se refiere a aquello que no puede ser reemplazable. Es decir, la repetición alude a tal singularidad que es imposible el intercambio o la sustitución. Por ello, y aquí introduce su primera referencia implícita a Hume, «los empiristas no se equivocan cuando presentan la idea general como una idea particular en sí misma, con la condición de agregarle la presunción de poder reemplazarla por cualquier otra idea particular ${ }^{22}$. Sin embargo, la repetición concierne a una conducta necesaria respecto al no reemplazamiento. Repetir es comportarse, insiste Deleuze, pero frente a algo singular y carente de equivalente.

Deleuze ofrece otra advertencia: la repetición no consiste en agregar a una primera vez una segunda, tercera o cuarta vez, sino que se trata de elevar esa primera vez a la enésima potencia ${ }^{23}$. La repetición, por tanto, es entendida como universalidad de lo singular, frente a la generalidad de lo particular. De este modo, sentencia que la repetición está en contra de la ley, la repetición es transgresión. La generalidad pertenece al orden de las leyes que determinan la semejanza de quienes se someten a ella. Sin embargo, la repetición no está fundada por la ley; es más, la ley pone de manifiesto cómo la repetición es imposible para los particulares, para los puros sujetos de la ley, porque los obliga a cambiar. Las constantes de una ley suponen la variación de una ley más general ${ }^{24}$.

Una vez aclaradas estas distinciones, es posible centrarse en el tema que nos ocupa, a saber, la influencia de la filosofía de Hume y, en concreto, del concepto de hábito en Diferencia y repetición. En la génesis de la subjetividad, el hábito, para Deleuze, juega un papel fundamental. El hábito se constituye como principio de la naturaleza humana, en la medida en que la subjetividad surge en la mente y la sobrepasa, constituyéndose como sujeto. El hábito, recordemos, opera cuando a la pura sucesión de casos se agrega una cierta progresión o tendencia. De este modo,

22 Ibidem, p. 115.

${ }^{23}$ Deleuze ejemplifica esto de una manera clara y visual tomando a Charles Péguy como referencia: es el primer nenúfar quien repite a todos los demás.

24 «... la repetición expresa [...] un elemento contra lo ordinario, una instantaneidad frente a la variación, una eternidad contra la permanencia. Desde todo punto de vista, la repetición es la transgresión. Pone la ley en tela de juicio, denuncia su carácter nominal o general, a favor de una realidad más profunda y artista». Deleuze, G., Diferencia y repetición, Amorrortu, Buenos Aires, 2012, p. 23. 
es posible introducirse en la repetición que entraña el hábito que, si bien requiere que esté más allá de la repetición de los casos, también debe posicionarse más acá de ellos para permitir su repetición. Esto es así porque los casos se repiten -en tanto que existe una tendencia que rija la repetición- en la medida en que el hábito ya se encuentra presente. A esta especie de círculo vicioso es necesario ańadir que, en lo que corresponde al placer, el hábito es una suerte de principio que lo complementa internamente. La repetición representa para el placer el reconocimiento de sus límites, génesis y fundamento. Dado que la repetición no debería retrotraerse de inmediato al placer, es preciso pensar en el carácter propio de la repetición como tal. El caso que se repite no cambia en virtud de la repetición:

La repetición no cambia nada en el objeto que se repite, más ella cambia algo en la mente que la contempla [...]. Se produce un cambio en la mente que contempla: una diferencia, algo nuevo en la mente ${ }^{25}$.

Deleuze insiste en que debemos distinguir, entre los objetos repetidos, un sujeto que se repite a través de ellos: «No hay una repetición sin repetidor, nada repetido sin alma repetidora ${ }^{26}$. Una repetición, por tanto, no debe nada a la otra, existe una independencia, una regla de discontinuidad que se establece en los distintos momentos de la repetición. Es en la mente donde se produce la alteración o cambio que permite retener una presentación hasta que aparezca la siguiente. Esto se produce en la imaginación, que genera una impresión interna capaz de contraer los distintos casos, generando una suerte de anticipación respecto a los que vienen. Lo que eso produce, en última instancia, es una síntesis temporal por la que la imaginación genera el vínculo que produce la presentación de impresiones sucesivas en la mente. Esta contracción no tiene como fin el establecer asociaciones o recuerdos, sino la formación de una síntesis primaria que tiene la capacidad de construir el presente viviente que retiene el pasado y anticipa el futuro. Aquí encontramos, precisamente, el carácter del hábito que se caracteriza por traer el pasado al presente, gracias a la contracción de los casos repetidos. Además, esta contracción es la que genera una tendencia que marca la anticipación respecto al futuro. Para explicarlo Deleuze recurre, una vez más, a Hume: ante la presentación reiterada de la conjunción $\mathrm{AB}$, la mente contrae la diversidad de casos y los lleva al presente, de tal forma que cuando se produce $\mathrm{A}$ la mente espera la presentación de B. La imaginación lleva a cabo una síntesis que da pie al flujo de impresiones, permitiendo, así, la retención en el presente del pasado:

La síntesis contrae los instantes sucesivos independientes los unos de los otros. Constituye, así, el presente viviente. Y el tiempo se despliega en este presente. A él pertenecen el pasado y el futuro; el pasado en la media en que los instantes prece-

25 Deleuze, G., Diferencia y repetición, p. 119.

${ }^{26}$ Ibidem, p. 124. 
dentes son retenidos en la contracción; el futuro porque la espera es anticipación en esta misma contracción ${ }^{27}$.

Este juego de aparecer y desaparecer incesante es el lugar en el que sitúa la regla de discontinuidad que caracteriza el modelo material de la repetición. Recordemos que una situación se piensa desde el punto de vista que la contempla; esto implica que, para la mente, los casos de la repetición se encuentran fundidos en una impresión que enlaza, de manera sucesiva, una presentación con las que le siguen. Ese movimiento trazado por la impresión dependerá de la fusión o contracción de los casos que se repiten para la mente.

Por tanto, la repetición no es tan siquiera pensable sin la contracción que permite entrelazar una presentación a otra, identificándolas como momentos de la repetición. Esto lleva a Deleuze a sentenciar que la repetición no existe más que para sí, pues es en la mente donde se establece el vínculo que permite identificar los momentos. Hablamos, entonces, de un para sí de la repetición que se refiere a cómo esta aparece para la mente.

En síntesis, la repetición depende de que retengamos las anteriores presentaciones del objeto y que las identificamos como momentos previos al actual. Será la contracción de la repetición la que trae el pasado al presente a partir de una retención que impide la desaparición de los momentos anteriores. Los siguientes momentos son traídos al presente y conforman la tendencia a la proyección al futuro. Deleuze llamará «síntesis pasiva» a la contracción que se produce en la imaginación y por la que surge la impresión en la mente: «La síntesis pasiva o contracción es esencialmente asimétrica: va del pasado al futuro en el presente; por consiguiente, de lo particular a lo general, y por ese camino, orienta la flecha del tiempon ${ }^{28}$.

Si bien Deleuze profundiza y matiza en Diferencia y repetición, estos planteamientos ya habían sido esbozados, como hemos visto, en Empirismo y subjetividad. Por ejemplo, cuando insiste en su preocupación por establecer el carácter del movimiento de las impresiones e ideas sin que esto produzca la operación de alguna facultad espontánea. A la par, expresa su interés en dilucidar el estatuto del hábito como principio de la naturaleza humana, forjada conforme a ciertos principios que actúan en la mente y lo convierten en sujeto. El hábito, explicará en Diferencia y repetición, establece la síntesis temporal inmanente al propio fluir de la mente. La contracción que deriva de esa síntesis establece el salto desde el movimiento de las impresiones hasta el conjunto de operaciones que lo convierten en sujeto. De este modo, a partir del concepto de síntesis pasiva, Deleuze trata de pensar la contracción inherente a la imaginación, que involucra una síntesis temporal, que hace posible la sucesión de los elementos de una repetición y la articulación con otros niveles de contracción:

27 Ibidem, p. 120.

${ }^{28}$ Ibidem, p. 120. 
Esta síntesis debe, desde todos los puntos de vista, ser nombrada: se trata de la síntesis pasiva. Constituyente, no es por ello activa. No está hecho por la mente, sino que se hace en la mente que contempla, precediendo toda memoria y toda reflexión. El tiempo es subjetivo, pero es la subjetividad de un sujeto pasivo ${ }^{29}$.

Esta síntesis temporal, antes bajo la exclusiva operación del hábito, será también propia de la vinculación inherente a la imaginación, lo que implica que hábito e imaginación están comprometidos en ese proceso que va de la mente a la subjetividad, a partir de la articulación de niveles de contracción cada vez más complejos.

En Empirismo y subjetividad el problema del hábito se establece como un problema independiente. Deleuze insiste en que, a partir de los elementos dados de manera discontinua, puede surgir una tendencia capaz de operar la síntesis temporal sobre la que los principios de la naturaleza humana llevan a cabo el conjunto de operaciones que convierten a la mente en sujeto. En el capítulo v de la citada obra, Deleuze analiza los principios y momentos por los que se constituye el sujeto en la mente. En la determinación de estos momentos, el hábito juega un papel clave. Si la mente se caracteriza por lo dado, recordemos, el sujeto constituye la emergencia de lo nuevo que, por tanto, no coincide con los datos de la mente y la rebasan. El papel del hábito en este rebasamiento (dépassement) será manifestado desde tres instancias distintas a medida que avanza el capítulo. La instancia que nos ocupa en este momento es la primera, la referida a la síntesis temporal que entraña. A este respecto, en Empirismo y subjetividad, a diferencia del análisis que he presentado en este apartado del hábito en Diferencia y repetición, la preocupación está centrada en poner de manifiesto el modo en que los principios de la naturaleza humana operan en el ámbito del conocimiento y la moral. Para esto se sirve del especial papel que juega, según Hume, la invención de reglas para el mundo moral y la creencia en el conocimiento. En ambos elementos, el hábito se comporta como principio de constitución. Se trata de avistar el modo en que se relacionan el hábito y la creencia que está en la base del conocimiento. La clave de esta relación, según Deleuze, es la tendencia que transmite la vivacidad de la impresión a la idea. Pero, además, insiste en que la invención está en el principio de emergencia de la espera que sirve como principio de acuerdo posible, generando, así, reglas de interés general. Es decir, en lo que respecta a la síntesis del tiempo, la creencia y la invención son dimensiones estrechamente ligadas, pues el hábito es la raíz constitutiva del sujeto, y lo que el sujeto es en su raíz es la síntesis del tiempo ${ }^{30}$.

Este conjunto de ideas será retomado por Deleuze en el segundo capítulo de Diferencia y repetición para explicar las tres síntesis del tiempo. En las dos primeras, la del hábito y la de la memoria, tendrán enorme relevancia las lecturas humeanas de Deleuze que han sido expuestas.

29 Deleuze, G., Diferencia y repetición, p. 15.

${ }^{30}$ Ibidem, p. 63. 
La síntesis del tiempo constituye el presente en el tiempo, esto no quiere decir que el presente es una dimensión del tiempo, sino que la síntesis constituye el tiempo como presente vivo. El pasado y el futuro son dimensiones del presente, «solo el presente existe», dirá Deleuze. Aunque sea posible concebir un presente coextensivo al tiempo - a contemplación al infinito de la sucesión de instantes, por ejemplo-, hay una imposibilidad física de ese presente; el presente se agota. Esto es así porque en la contracción siempre opera la calificación de un orden de repetición según elementos o casos. El presente tiene una cierta duración que varía según individuos, especies u organismos ${ }^{31}$. La primera síntesis del tiempo, la del hábito, constituye el tiempo como presente que pasa. El presente se mueve sin salir de sí mismo, tal es la paradoja del presente que señala Deleuze; constituir el tiempo, pero pasar en ese tiempo constituido. El hábito supone la fundación del tiempo; este es, en palabras de Deleuze, el suelo móvil ocupado por el presente que pasa. Siendo el pasar la propia pretensión del presente. Pero ¿qué es lo que hace pasar al presente? Aquello que fundamenta al tiempo, a saber, la memoria. La memoria, como síntesis activa, descansa sobre el hábito, es decir, se apoya en la fundación; sin embargo, esto no la constituye. Pues si esta se funda sobre el hábito, no debe estar fundada por él, sino por una síntesis pasiva distinta. La propia síntesis pasiva del hábito remite a otra más profunda que pertenece a la memoria:

El hábito es la síntesis originaria del tiempo, que constituye la vida del presente que pasa; la memoria es la síntesis fundamental del tiempo, que constituye el ser del pasado (lo que hace pasar el presente) ${ }^{32}$.

La segunda síntesis, entonces, hace pasar el presente y advenir otro. De este modo, el pasado se encuentra entre dos presentes, el que ha sido y aquel con respecto al que es pasado. No se trata de que el pasado sea un antiguo presente, sino aquello en lo que se enfoca el presente (pasado general). El hábito comprendido como los instantes sucesivos contraídos en el presente actual de cierta duración es clave para entender que esos instantes forman una particularidad que se corresponde con un pasado inmediato que pertenece al presente actual. Por su parte, en la reproducción de la memoria, el pasado se vuelve general y no particular, como mediación de los presentes. Esto quiere decir que el pasado en general es el elemento en el que puede enfocarse cada antiguo presente y que cada uno de ellos se encuentra representado en el actual. En este punto, Deleuze recurre de nuevo a Hume para explicar que los límites de esta representación están determinados por las relaciones de semejanza y contigüidad, es decir, por las reglas de asociación que había expuesto Deleuze en los primeros capítulos de Empirismo y subjetividad y a las que dediqué los puntos

31 «Dos presentes sucesivos pueden ser contemporáneos de un mismo tercero, más extendido por el número de instantes que contrae. Un organismo dispone de una duración de presente, de diversas duraciones de presente, según el alcance natural de contracción de sus almas contemplativas». Deleuze, G., ibidem, p. 129.

32 Ibidem, p. 133. 
iniciales de este trabajo ${ }^{33}$. Esto se explica porque para que el antiguo presente pueda ser representado, debe parecerse al actual, disociándose en presentes parcialmente simultáneos de diversas duraciones, es decir, contiguos unos a otros, pero también al actual.

El antiguo y el actual presente no deben ser entendidos como instantes sucesivos en la línea temporal, sino, más bien, que el actual contiene necesariamente una dimensión más por la cual re-presenta al antiguo y a sí mismo. El presente actual, explica Deleuze, no está tratado como el objeto futuro de un recuerdo, sino como lo que se refleja, al tiempo que forma el recuerdo del antiguo presente.

Hábito, memoria y síntesis temporal juegan un papel fundamental en el planteamiento deleuziano de la repetición. Lo que pretende este último punto es poner de manifiesto las influencias y resonancias de la primera obra de Deleuze, dedicada a Hume, en una de sus tesis Diferencia y repetición. Su lectura del hábito, la memoria y los principios de asociación se han erguido como pilares básicos en la comprensión del concepto de repetición. El hábito, como primera síntesis del tiempo, constituye el tiempo como presente vivo en una fundación pasiva de la que dependen el pasado y el futuro. La memoria, como segunda síntesis, constituye el tiempo como pasado puro desde el punto de vista de un fundamento que hacía pasar el presente y advenir otro. Sumar la tercera síntesis - que se refiere al presente como actor destinado a borrarse, como porvenir-implica la capacidad de revelar el pasado, el presente y el futuro como repetición. El presente es repetidor, el pasado es la repetición misma y el futuro es lo repetido.

Una filosofía de la repetición pasa por todos los estadios, condenada a repetir la repetición misma. Pero a través de esos estadios asegura su programa: hacer de la repetición la categoría del porvenir, servirse de la repetición del hábito y de la memoria (2012: 151) ${ }^{34}$.

Deleuze y su comúnmente olvidada lectura de Hume nos anima a entender que no se trata de sonsacar la diferencia a la repetición, sino de hacer de la repetición la diferencia en y por sí misma. Pues, a partir de la interpretación deleuziana del eterno retorno nietzscheano, lo que se repite, lo que vuelve, es diferencia.

Recibido: septiembre de 2020; ACEPTADO: diciembre de 2020

${ }^{33}$ La importancia del asociacionismo reside en haber fundado toda una teoría de los signos artificiales sobre esas relaciones de asociación». Ibidem, p. 134.

34 Ibidem, p. 151. 